\title{
A Review of the Empirical Evidence of the Healthcare Benefits of Personal Health Records
}

\author{
D. Kalra, B. Fernando \\ Centre of Health Informatics and Multiprofessional Education, University College London, London, \\ United Kingdom
}

\begin{abstract}
Summary
Objectives: This literature review sought to identify the established evidence of the health and healthcare benefits (or harms) from the use of electronic personal health records (PHRS) and PHR systems.

Methods: The definition of a PHR published in ISO 14292 was used to scope this review and the search strategy. Publications were included if the introduction of a PHR was the primary intervention, and if its evaluation met one of the Cochrane EPOC Group criteria. Studies were excluded if they only reported the design or basic user acceptance of a PHR system without an assessment of its impact on individuals and/or their health care. The impacts were classified according to the six aims of 21st-century health care defined by the US Institute of Medicine.

Results: Searches were conducted in PubMed in December 2012. Out of 741 papers that met our initial search criteria, 31 were retained after title and abstract screening. After full paper review 5 studies were found to report original evidence of impact. Of these, three reported beneficial impacts on effectiveness, one on patient centredness, and one study reported impact on both aims. No harmful effects were reported.

Conclusions: Although this literature review did identify some evidenced benefits from the use of PHRs and systems, our main observation is that there are very few studies published that seek to formally evaluate impact. The majority of publications we screened documented designs or basic user acceptance. Further investment in evaluation is needed to inform the evolution of this field.
\end{abstract}

\section{Keywords}

Personal health records, evaluation research, review, evidence-based health care, empirical research

Yearb Med Inform 2013:93-102

\section{Introduction}

It is not a new or recent phenomenon for individuals to keep personal records of aspects of their health and wellness. For generations this kind of information has been documented in diverse ways such as the growth of children marked in pencil on a kitchen wall, pocketbooks documenting curious symptoms to be reported later when visiting the doctor, a diet book or calorie counter kept during a weight reduction program. Over the past couple of decades, inevitably, some of this documentation has migrated from paper charts and books to word-processed documents, desktop applications, mobile applications and, most recently, multi-device access through clouds, just as our diaries and address books have. This has enabled these tools to become richer in knowledge content and function, offering ways of reviewing historic trends and future projections, links to relevant online information and reminder functions.

However, interest in personal health records, amongst patients, healthy citizens, healthcare professionals, industry and insurers has grown in recent years in ways that go beyond the natural evolution of consumer electronics. Citizens in general are paying a greater attention to their health, as well as their fitness, and this has led to a proliferation of applications and services to support people in capturing, monitoring, comparing, understanding and sharing their personal health/fitness status. Health care providers and insurers have begun to anticipate that engaging citizens in the self management of chronic diseases, and preventive health strategies, will contribute to improved clinical outcomes, a reduced or delayed onset of complications, the prevention of disease and to greater patient satisfaction with health care services. In recent years a number of health care providers have developed a patient portal, not only to provide direct online access to specific healthcare services such as appointments and test results, but also to enable individuals to use portal applications to track elements of their own health status. Well-known examples of patient portals include Kaiser Permanente's MyHealthManager [1] and the US Veterans Administration's My HealtheVet [2].

There is now increasing importance given to patient empowerment and self-management through strategic initiatives such as the European Commission's European Innovation Partnership on Active and Healthy Ageing [3], which will sponsor several large scale demonstrators of integrated care and patient inclusion innovations, and programmes such as the NHS Expert Patients Programme [4] which offers patients with long term conditions a package of educational courses designed to increase their confidence and capability with managing their health.

Increasing investments are being made in Personal Health Records (PHRs) by health care providers, and by citizens (the latter, for example, purchasing apps from mobile application stores). The PHR market is predicted to expand rapidly (estimated at over 400 million USD by 2015)[5]. It is important that such investments target the health and wellness management functions that will deliver benefits to health and healthcare, and avoid any potential harm to patients. Future PHR system developments and wide scale adoption therefore need to be informed by the emerging evidence of impact.

This paper seeks to answer the question of whether we have yet established empirical evidence of any of the anticipated health gains through the use of personal health records. 


\section{Definition and Scope of a Personal Health Record}

There is a rapidly expanding diversity of Personal Health Record systems in existence or anticipated, meeting different kinds of need to keep subjects of care informed and engaged in health and social care, or to enable individuals who are not needing or seeking healthcare to keep track of their level of fitness, manage prevention or monitor health status. The concept of a PHR is therefore not a singular entity but rather encompasses a spectrum of possible information repositories and services, which is rapidly expanding. The PHR is an area of innovation by information and technology (ICT) companies, wellness organisations, health insurers and healthcare providers. At its most comprehensive, a PHR may encompass health, wellness, development, welfare and health concerns; at a chronological breadth which embraces history of past events, actions and services; tracking and monitoring of current health or activities; and goals and plans for the future. Some PHRs will have a general health overview focus; others may target a specific healthcare or wellness function e.g. a diabetes management record or a personal fitness record. Simple examples of PHRs include self-contained mobile phone applications that track a personal diet or exercise history. An individual may choose to have one single overall PHR or multiple activity-driven PHRs, or a combination of both.

The 2004 Markle Foundation report "Connecting Americans to their Health Care" defines the personal health record as:

"An electronic application through which individuals can access, manage and share their health information, and that of others for whom they are authorized, in a private, secure, and confidential environment." [6]

Tang et al report on a 2005 AMIA symposium on personal health records, which took their working PHR definition from the Markle Report [7]. This symposium considered many possible functions of personal health records, including access to credible health information, tracking of chronic diseas- es, improved communications, continual electronic interaction between patients and physicians, but recognised that evidence is (rather was) yet lacking to support their potential benefits.

Pagliari, Detmer and Singleton published a concept paper discussing the range of PHR functions, the possible benefits to individuals and to healthcare, and early PHR initiatives in the UK, in 2007 [8]. Their working definition of a PHR was also taken from the 2004 Markle Foundation report. The key PHR functions they identified were:

- access to a provider's electronic clinical record;

- personal health organiser or diary;

- self management support;

- secure patient-provider communication;

- links to static or interactive information;

- links to sources of support;

- capture of symptom or health behaviour data.

Their investigation did not seek to identify the benefits or evidence of outcomes from the use of PHRs.

The most recently developed, and internationally endorsed, definition of a personal health record was published by the International Organization for Standardization in 2011 (ISO 14292) [9].

"A Personal Health Record of an individual is a representation of information regarding or relevant to the health, including wellness, development and welfare of that individual, which may be stand-alone or integrating health information from multiple sources, and for which the individual, or the representative to whom the individual delegated his or her rights, manages and controls the PHR content and grants permissions for access by and/or sharing with other parties."

PHRs have some similarities with electronic Health Records (EHRs), which ISO defines as a "logical representation of information regarding or relevant to the health of a subject..." [10]. Both consider the scope of health very broadly, according to the WHO 1946 definition [11]. However, wellness part of the scope of a PHR according to ISO - is an active process of becoming aware of, and making choices toward a more successful existence, which goes further than the WHO definition of health.

Healthcare organisations and healthcare systems are accountable for the content of EHRs that they create and control. The key "definitional" distinction between the PHR and the EHR is that, in the former, the individual who is the subject of the record is the key stakeholder determining its content and with rights over that content. This might be through the subject personally entering the content, or by the subject authorising one or more parties or systems to contribute to the PHR, or by the subject authorising the creation of a PHR on his or her behalf by an organisation or person whose anticipated purpose is considered relevant and trustworthy by the subject. The individual always retains rights over the information content held within a PHR, including the ability to delegate those rights to others (especially in the case of minors, the elderly or the disabled). However, functionally, there may be many similarities between a PHR application and an EHR portal offered by a provider to its patients.

The ISO 14292 definition does not imply that the subject is primarily responsible for managing the repository, nor that he or she is the legal data processor or legal owner of the record system on which it is held. Other organisations may perform some or all of these roles on behalf of the individual, usually through a formal agreement or product licence.

ISO 1429 recognises the diversity of PHR market offerings, and that this is an active area of innovation. The above definition therefore focuses on the PHR's key distinguishing characteristics rather than its features. The Report also specifies six dimensions along which any given PHR solution might be classified.

1: Scope of the information: the kinds of information and information sources reflected in the PHR content (e.g. information about health and wellness, about contact with carers and support organisations, copies of health summaries from an EHR, condition-specific).

2: Control over the information: the extent of the authority of the subject of the 
record over the policies that define who can access and modify his or her PHR (e.g. provide PHR access to specified persons, specify access to particular entries or sections of the PHR, control if that access is read only etc.), and thereby determine the extent to which the subject wishes to assert the privacy of their PHR content.

3: Data processor: the party who acts as the data processor of the repository, in a legal sense (for example, registering under data protection legislation) and also operationally (e.g. the individual, the vendor of a PHR product, a trusted third party, a healthcare provider, employer).

4: Repository auditability: the extent to which the PHR manages its content in ways that meet the kinds of legal requirement expected of an EHR, for example, as specified in ISO 18308 (which may influence the extent to which the information in it is considered trustworthy for use by health professionals or integration with EHRs). Compliance with such requirements will require the use of information security measures to protect the PHR from inappropriate access and modification.

5: Interoperability and communication: the extent to which the PHR is able to be accessed remotely and is able to share information with other pertinent repositories such as EHRs (this relates to the ability of the repository to support such interoperability and communications, rather than whether they are in operation for a particular PHR or repository).

6: Technical architecture: the way in which the PHR has been implemented, in terms of the platform and distribution architecture supported (e.g. stand alone applications with integrated databases, on a computer workstation or mobile device, able to synchronise data between more than one device, communicate with a web service to enable access from anywhere).

The goal of these dimensions is to convey the diversity of what might be considered a PHR, and help to formalise how PHRs offerings can be compared.

PHRs will increasingly be combined with smart applications and decision support tools into Personal Health Systems (PHS) [12]. These may include the provision of person- alised education and guidance (including real-time reminders and prompts) to assist individuals with the self-management of long term conditions and/or with lifestyle guidance such as smoking cessation. Innovations in PHS have been a key topic within recent European Health ICT research calls for proposals [13]. As these systems become more sophisticated, with the aim of reaching a widening range of patients and healthy individuals, it will be important that the applications are easy to use, and capable of being used consistently and accurately by persons of different backgrounds and skills (usability). This will be particularly important as PHS enter the arena of multi-morbidity, supporting an individual in the care of more than one condition.

It should be noted that some personal health systems, such as portable and wearable medical devices, might contribute data to a PHR, but are not in themselves PHRs. Many Web sites provide public information about health and health services to inform individuals about self care and clinical care options; however, most of these are not, or do not contain, personal health records. Social networking sites enable individuals to share personal health information with others, usually for mutual support and occasionally to develop expert resources for others to access. Although containing personal health information, these sites do not function as cumulative personal records and are not under the control of the members themselves - as individuals. These are therefore also not considered as PHRs for the purpose of this review. Rigby gives a useful discussion of various forms of personal health systems and services [14].

\section{Tethering between PHRs and EHRs}

Health information within a PHR may be purely for use by the individual him or her self, or may be shared with healthcare professionals and others, such as family members. The inclusion of EHR extracts within a PHR, for example laboratory reports or discharge summaries, is a desired feature of a comprehensive PHR but in order to preserve data integrity, might only be annotated with comments by the individual and not edited.

Reciprocally, many healthcare providers are developing portals and applications that enable patients to directly access information and services, including views on their EHRs. These portals may offer a means for patients to schedule elements of their healthcare, view test results, put questions to their healthcare providers, and sometimes offer a richer interaction - for example to collaborate in the monitoring and management of a long term condition. These EHRs are in effect evolving to include some PHR like behaviours, but rarely offer the patient genuine autonomy over their content. These kinds of patient or citizen portals have therefore not been considered as PHRs for the purposes of this review.

The term "tethered" is sometimes used, especially in the US, to denote certain kinds of personal health record that are offered by a patient's health care provider. Tethered records are themselves not a singular entity, but cover a spectrum of different offerings. Their common characteristics include: the system is developed by or for the health care provider, who is the data controller; the patient is provided access, but in other ways the provider retains authority over access control; many of the functions offered by the application will support the patient in accessing the healthcare services offered by the provider; the primary information content comes from the EHR of the patient as held by the provider; offering read only access to parts of the EHR is sometimes considered the end in itself, and is confusingly sometimes termed a personal health record. As discussed earlier, for the purposes of this review a personal health record must give individuals control over content and access. The term "tethered" was therefore deliberately not included within our search strategy, and any evaluations of personal health records that were found to really be tethered EHRs were only included in the review if the system also provided genuinely personally-controlled features including some capacity for personally determined content (i.e. not just for entering monitoring data under the direction of a clinician). 


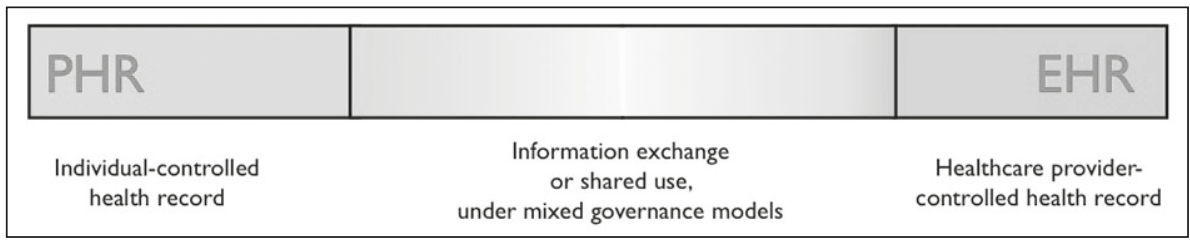

Fig. 1 The EHR-PHR spectrum (reproduced from ISO 14292:2011)

- AND an empirical evaluation reported on one or more benefits obtained from the use of the PHR, or any harmful effects identified, and meeting the $\mathrm{Co}$ chrane's EPOC Group criteria, namely: a randomised controlled trial, controlled clinical trial, controlled before-and-after study or interrupted-time-series [17].

\section{Exclusion Criteria}

Some examples of a tethered PHR are primarily a patient access portal to view the healthcare provider's EHR, and possibly to undertake some healthcare service functions such as appointment scheduling, requesting medication refills and email. For the purposes of this review, tethered systems were considered to be within the central portion of Figure 1, and included in this review, if they included features for patients to enter personal health information that were personally directed, and that these entries were used as part of continuity of care.

\section{Methods}

The search strategy was developed by iteratively scoping the literature, through discussion with experts, and from previously published search strategies, in particular [15]. A list of terms variously used to describe personal health records research in PubMed has recently been collated by Kim et al, and also informed this search strategy [16].

\section{Search Strategy}

The following search strategy was performed using the PubMed advanced search online tool, on 27 December 2012.

("personal electronic health record" OR "personal electronic health records" OR electronic personal health record OR electronic personal health records OR "personal health record" OR "personal health records" OR "personal health system" OR "personal health systems")

A comprehensive search strategy for types of evidence was also developed, using terms that filtered for case-control studies, cohort studies including prospective cohort studies, cross-sectional studies, clinical trials, epidemiological studies, qualitative studies and systematic reviews. However, applying this strategy with an AND clause plus the above filter was found to reduce the total number of positive matches too greatly. It was therefore decided to perform a manual filtering for such evidence using the matches returned on the above search strategy.

No date filter was applied.

\section{Screening Criteria}

Title and abstract screening was undertaken on the basis of the criteria defined below. DK undertook the initial screening. Any uncertain decisions were referred to BF, and only rejected if both reviewers concurred. Full paper screening was undertaken using these same criteria and performed independently by DK and BF. Data from empirical studies that met the selection criteria were abstracted into an evidence table; findings were then first descriptively summarised and then thematically synthesised according to the kind of outcome examined for.

\section{Inclusion Criteria}

- a specified PHR or PHR system conforming to the ISO 14292 definition, as the primary intervention of the research, in particular that individuals had control over (at least some elements of) the PHR content including the ability to contribute to it in personally determined ways;

- AND its reported use by at least one target community, patient group, healthcare setting;
- descriptions (without evaluation) of in-progress development of PHR systems or their supporting applications, interoperability or security and privacy features; publications describing relevant novel products and services for which evaluations had not yet been performed;

- feasibility or proof of concept studies demonstrating technical success and/or usability and patient or clinician acceptance of a PHR or application;

- evidence of the use of a PHR, and the collection of data within it, including user and usage profiling, without any evidence of a benefit derived from those data;

- feasibility or quality assessments of the potential for PHR data to support a quality improvement or clinical outcome, which did not seek to corroborate this through use of an actual PHR;

- benefits from read-only access to a provider EHR, or from patient monitoring $\operatorname{logs}$ in which the information to be collected was directed by a healthcare provider (i.e. not conformant to ISO 14292);

- social networking sites that primarily focus on building a community or a shared resource rather than person-centric health records;

- feasibility assessments or actual secondary uses of PHR data for: research, epidemiology, public health, health services research, health service evaluations or reimbursements;

- complex interventions in which impact from the introduction of a PHR was not capable of being isolated from other causes of the impact (e.g. educational programs and service delivery changes in parallel to the introduction of a new PHR). 
The finally selected publications were thematically grouped according to the kind of outcome reported. The outcomes were mapped to the six aims of improvement for 21 st century healthcare systems in the Institute of Medicine's (IOM) Crossing the Quality Chasm report, i.e. improvements in safety, effectiveness, patient-centeredness, timeliness, efficiency or equitability of the care delivered to patients [18], but recognising that for some such as safety and equity it may have only been possible to look for proxy measures. It was recognised that some person-centred benefits might be outside the scope of these six aims, and if encountered would be listed separately.

\section{Results}

Initial searches resulted in 742 papers for consideration, reduced to 741 after removal of a duplicate. After title and abstract screening, 31 papers satisfied our inclusion criteria (see Figure 2 for the PRISMA diagram).

After full paper review only five publications were found to meet our criteria. The majority of those, which were rejected, had offered patient access to an electronic health record that was termed a personal health record but in fact offered little or no capability for the patient to contribute personal health information. Several studies had collected usage information to indicate which portions of a provider record has been accessed, but had not identified if these accesses had resulted in any value. Other publications had implied from the abstract that some beneficial outcome had been identified, but on studying the full paper it became apparent that these benefits were conjectural (for example asking patient how they thought their personal health record might be useful). Of the five publications, which met the selection criteria, four reported outcomes that corresponded to the IOM aim of effectiveness, of which two also reported improved patient centeredness. One study reported an efficiency gain, albeit qualitatively. There were no reported benefits that fell outside of these categories. The findings from these five publications are summarised below, and reported in detail in Table 1.

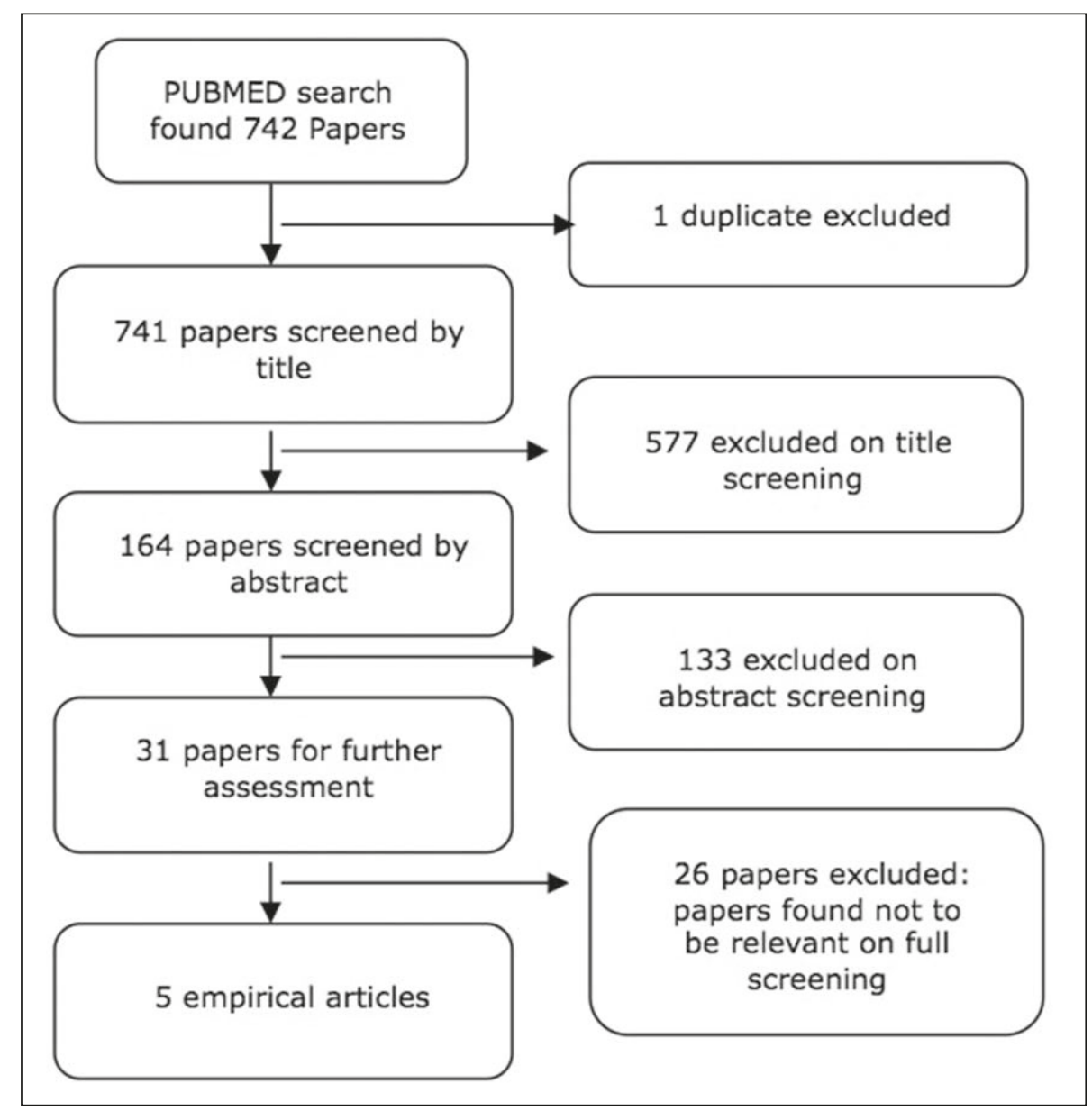

Fig. 2 PRISMA flow diagram

\section{Outcome Type: Effectiveness and Patient-centeredness}

Nagykaldi et al report the results of a cluster-randomised controlled trial in Oklahoma, USA of patients attending eight physician practices [19]. The practices were matched for physician demographics and socio-demographic factors of their patient populations, into two groups of four. A web based patient portal and PHR was provided to recruited patients attending four of the practices. This provided features to manage a history of preventive health care, tracking of personal risk factors and personal preferences, the generation of a personalised wellness plan, tracking of vital signs and test results, a symptom diary, the ability to manage medication lists and immunisation records, and secure messaging with physi- cians. A total of 384 patients were recruited and completed the 12 month study. Patients in intervention practices were asked to update their risk factors, allergy and contraindication profile, and personal preferences before each clinic visit, to generate personalised care recommendations and discuss these with their physicians. Patients were surveyed by questionnaire at baseline and after 12 months, and patient centeredness was measured by an adapted Consumer Assessment of Health Care Providers and Systems instrument. $73 \%$ of patients (out of the 280 in the intervention group) updated their initial records, 128 patients updated immunizations, 117 patients documented preventive services, 77 recorded risk factors. Intervention group participants received $84.4 \%$ of all recommended preventive services; in the control group only $67.6 \%$ 
Kalra et al.

Table 1 Evidence table summarising the key findings from the five papers examined in this review

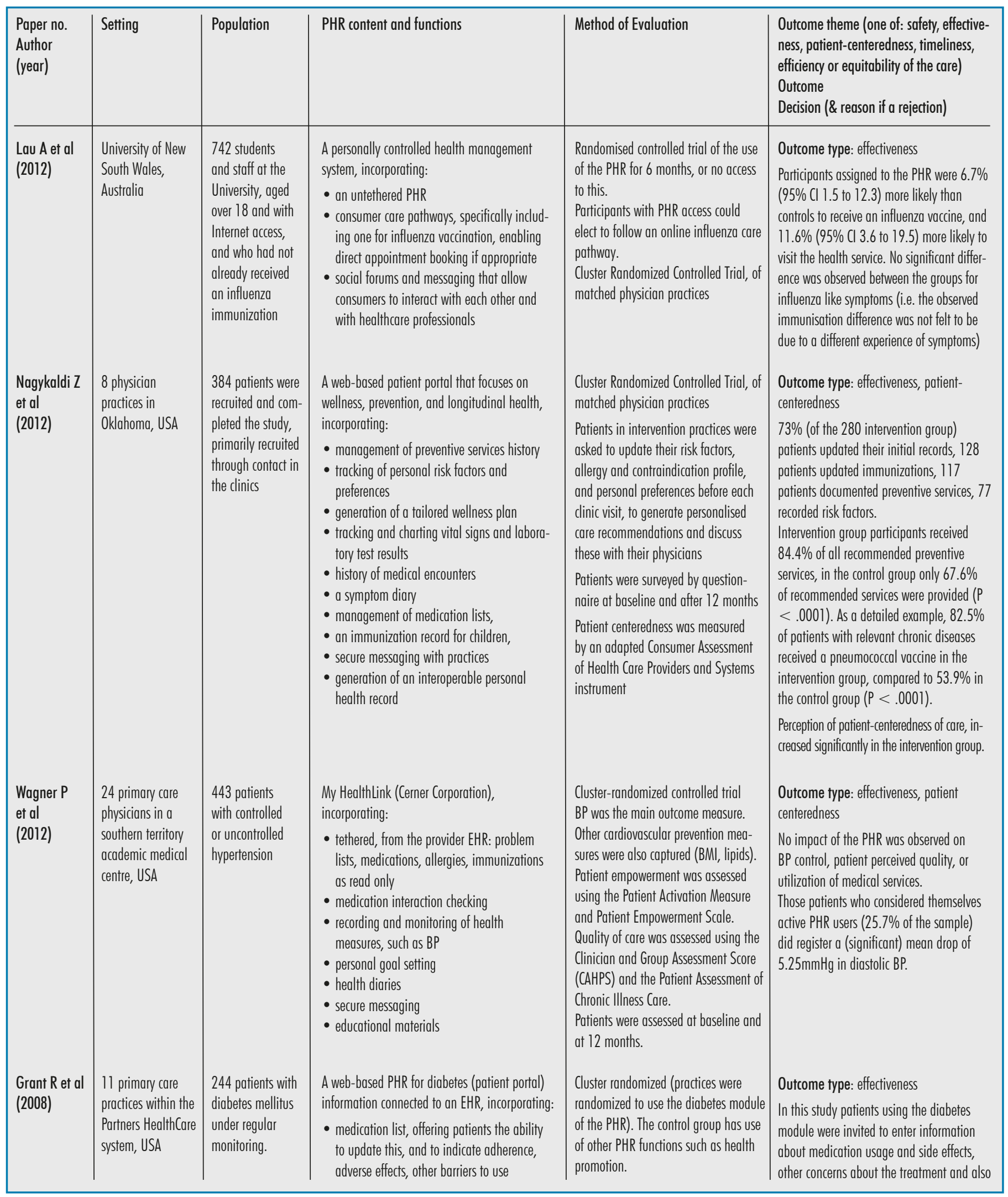




\begin{tabular}{|c|c|c|c|c|c|}
\hline $\begin{array}{l}\text { Paper no. } \\
\text { Author } \\
\text { (year) }\end{array}$ & Setting & Population & HR content and functions & Method of Evaluation & $\begin{array}{l}\text { Outcome theme (one of: safety, effective- } \\
\text { ness, patient-centeredness, timeliness, } \\
\text { efficiency or equitability of the care) } \\
\text { Outcome } \\
\text { Decision (\& reason if a rejection) }\end{array}$ \\
\hline $\begin{array}{l}\text { Kim E et al } \\
(2005)\end{array}$ & $\begin{array}{l}\text { Evereft Housing } \\
\text { Authority, which } \\
\text { provides housings } \\
\text { for low- income } \\
\text { families and } \\
\text { elderly or disabled } \\
\text { populations. } \\
\text { Adult residents } \\
\text { of the Broadway } \\
\text { Plaza, } \\
\text { Washington, USA }\end{array}$ & $\begin{array}{l}\text { Low income } \\
\text { families. } \\
24 \text { volunteers } \\
\text { were recruited out } \\
\text { of an elderly res- } \\
\text { ident population } \\
\text { of } 180\end{array}$ & $\begin{array}{l}\text { - view of most recent test results and vital } \\
\text { signs } \\
\text { - ability to enter concerns about present } \\
\text { monitoring and treatments } \\
\text { - } \\
\text { - Demographics } \\
\text { record system (PHIMS) } \\
\text { - a list of contacts and power of attorney for } \\
\text { healthcare } \\
\text { - insurance information } \\
\text { - healthcare providers and clinics } \\
\text { - past and current medical history including } \\
\text { health problems } \\
\text { - lab tests, } \\
\text { - immunizations, } \\
\text { - allergies, } \\
\text { - operations } \\
\text { - medications } \\
\text { - patients could add the reasons for taking } \\
\text { each medication item, and how helpful } \\
\text { each medication was proving to them } \\
\text { Residents determined the content of the PHR, } \\
\text { and could use this to combine information } \\
\text { about care from multiple providers. Elderly } \\
\text { residents were helped to complete their PHR } \\
\text { by visiting nursing students. } \\
\end{array}$ & $\begin{array}{l}\text { The primary goal of this study was } \\
\text { to observe impact on the clinical } \\
\text { management of diabetes in primary } \\
\text { care practices, after } 12 \text { months use of } \\
\text { the diabetes PHR module. } \\
82 \text { patients submitted updates to their } \\
\text { PHR in the intervention group } \\
\text { Usage statistics and a questionnaire } \\
\text { completed by only } 12 \text { (50\%) of the } \\
\text { PHR users. }\end{array}$ & $\begin{array}{l}\text { concerns and wishes related to their care plans. } \\
\text { Many of the patients who updated their PHR } \\
\text { indicated preferences for more stringent control } \\
\text { of their blood glucose ( } 51 \% \text { of patients), BP } \\
(32 \%) \text {, or lipids ( } 28 \%) \text { within their care plans. } \\
\text { These users of the diabetes module were more } \\
\text { likely to have changes to their medication made } \\
\text { at the next clinic visit to more intensively target } \\
\text { control of one or more physiological parameters. } \\
\text { The study concluded that pre-visit use of a } \\
\text { PHR linked to an EHR may increase the rate of } \\
\text { diabetes-related medication adjustments. } \\
\text { Outcome type: efficiency } \\
4 \text { out of the } 12 \text { of participants who completed } \\
\text { a questionnaire stated that they had taken their } \\
\text { PHR (usually in printed form) to a clinic where } \\
\text { the specialist had found it helpful and needed } \\
\text { less time to gather information from the patient. } \\
\text { Resident3: "....Went to specialist, Nephrologist, } \\
\text { they copied my PHIMS and I did not have to } \\
\text { fill out forms, they were very impressed with } \\
\text { e-record and used it."It would save time for the } \\
\text { healthcare provider. } \\
\text { No evidence was sought of improvement in } \\
\text { care as a consequence of using or sharing this } \\
\text { unified PHR. }\end{array}$ \\
\hline
\end{tabular}

of recommended services were provided $(\mathrm{P}<.0001)$. As a detailed example, $82.5 \%$ of patients with relevant chronic diseases received a pneumococcal vaccine in the intervention group, compared to $53.9 \%$ in the control group $(\mathrm{P}<.0001)$. Perception of patient-centeredness of care increased significantly in the intervention group.

Wagner et al also conducted a cluster randomised controlled trial amongst primary care physicians, in a US southern territory [20]. This PHR, My HealthLink (developed by Cerner Corporation), provid- ed a tethered PHR comprising problem lists, medications, allergies and immunisations in a read only form, the ability to record and monitor health measures such as BP, to set personal goals and maintain a health diary. The primary objective of this study was to examine the impact on BP control of access to the PHR. BP was the main outcome measure, but patient empowerment was also assessed using the Patient Activation Measure and Patient Empowerment Scale. Quality of care was assessed using the Clinician and Group Assessment Score (CAHPS) and the Patient Assessment of Chronic Illness Care. No impact of the PHR was observed on BP control, patient perceived quality, or utilization of medical services. Those patients who considered themselves active PHR users (25.7\% of the sample) did register a (significant) mean drop of $5.25 \mathrm{mmHg}$ in diastolic BP. It is, however, difficult to determine if these active users were generally more motivated towards achieving good control of their blood pressure, and if this observed reduction was genuinely a PHR effect. 


\section{Outcome Type: Effectiveness}

Lau et al report on a study involving 742 students and staff at the University of South Wales in Australia, on the impact of a personal health record system on influenza vaccine uptake [21]. The personally controlled health record was untethered to any provider electronic health record system. Its key feature relevant to this study was the incorporation of consumer care pathways, which could be personalised and used interactively, including the capability to directly book appointments with the university health service if needed. Participants were randomised to have access to this PHR, including an influenza care pathway, for six months prior to the control group. Participants assigned to the PHR were $6.7 \%$ (95\% CI 1.5 to 12.3 ) more likely than controls to receive an influenza vaccine, and $11.6 \%$ (95\% CI 3.6 to 19.5 ) more likely to visit the health service. No significant difference was observed between the groups for influenza like symptoms (i.e. the observed immunisation difference was not felt to be due to a different experience of symptoms).

In 2008 Grant at al reported a cluster randomised trial in 11 primary care practices that were part of the Partners HealthCare System in the US [22]. 244 patients with diabetes who were actively being reviewed on a regular basis for monitoring were recruited to the study. Practices were randomised to offer access to a newly developed diabetes module of a pre-existing personal health record system, which was available across all practices. Recruited patients of the control practices were able to access other basic PHR functions such as health promotion. The objective of the study was to observe the impact on diabetes management after 12 months of use of the diabetes module. Patients using that new module were invited to enter information about medication usage and side effects, other concerns about the treatment and also concerns and wishes related to their care plans. Many of the patients who updated their PHR indicated preferences for more stringent control of their blood glucose (51\% of patients), BP $(32 \%)$, or lipids $(28 \%)$ within their care plans. These users of the diabetes module were more likely to have changes to their medication made at their next clinic visit, in order to more intensively target control of one or more physiological parameters. The study demonstrated that pre-visit use of a PHR linked to an EHR increased the rate of diabetes-related medication adjustments.

\section{Outcome Type: Efficiency}

In $2005 \mathrm{Kim}$ et al conducted a small study of an untethered PHR with volunteer residents of a housing association property, largely comprising elderly residents [23]. 24 volunteers used the PHR, which provided the ability to document demographics, a provider contact list, insurance information, past and current medication, health problems, laboratory tests, immunisations, allergies, operations. Residents could add information about medications including why they were being taken, if they had any difficulties or adverse effects from taking them, and how helpful they found the medication items. The residents determined the content of the PHR and importantly could use it to combine information about care received from multiple health care providers. Because of the potential difficulty of elderly residents in completing this PHR, they were supported in data entry by visiting nursing students. Only 12 of the volunteers completed the end of study survey. 4 out of the 12 of participants who completed a questionnaire stated that they had taken their PHR (usually in printed form) to a clinic where the specialist had found it helpful and needed less time to gather information from the patient. A resident quoted in the paper stated:

"....went to specialist, Nephrologist, they copied my PHIMS and I did not have to fill out forms, they were very impressed with e-record and used it."

No evidence was sought of improvement in care as a consequence of using or sharing this unified PHR.

\section{Discussion}

The findings of this research echo the observations of previous literature reviews on this subject: that very few empirical studies have been undertaken to look specifically for clinical and/or patient outcomes, and that these have struggled to demonstrate statistically significant impact [7, 16, 24, 25].

Even though paper-based patient health records have been used for decades to support clinical shared care and for informing patients, there is also a lack of evidence of any specific benefits from these. As an example, Gysels et al searched the literature specifically for trials of the use of patient-held records in cancer care, and identified 13 studies evaluating such booklets, none of which demonstrated a significant impact on clinical care, communication, or patient satisfaction [24]. A recent literature review of personal health records, undertaken by Archer et al, up to March 2010, focused specifically on electronic personal health record systems and found only three randomised controlled trials and none demonstrating any improvement in actual health outcomes, although users reported perceiving value in having access to more information [25]. Because of the wide spectrum of potential roles of personal health record systems within health and wellness management we did pilot some other search terms, but found that these did not yield further relevant publications. It is nevertheless possible that our search strategy missed some publications that were not indexed as personal health records or systems.

Although we did not specifically look for this, our literature review did reveal examples of the value from patient access to their provider's electronic health record. Schnipper et al demonstrated that patients could usefully correct their EHR information (such as medication lists) if they are given online access to this information [26]. Turvey et al found that veterans using the My HealtheVet online electronic health record (a tethered PHR) had chosen to share their medication lists with non-VA healthcare providers [27]. It is recognised that medication interactions are an important patient safety issue, and it is therefore feasible that correct and shared medication lists might contribute to improved patient safety, but these studies did not seek to identify a measurable safety impact. 
A limited extension to the fully tethered PHR is the ability for patients to enter disease monitoring readings. Although this review deliberately did not search for evidence of this nature, it is recognised that EHR systems that allow patients to enter monitoring data such as blood glucose can result in better control, for example, Tenforde et al [28]. However, it is difficult in such studies to isolate and control for the commitment of individuals to have a better disease control from their commitment to providing monitoring readings online. Tenforde et al conclude: "this is an association study in which we cannot infer causality between PHR adoption and use and quality of diabetes management."

When considering the wellness and preventive health end of the spectrum, it is admittedly difficult to demonstrate outcomes in the short or medium term. For example, Krogsbøll et al have recently conducted a systematic review looking for evidence of concrete morbidity and mortality outcomes from general health checks in adult patients, and failed to find any evidence of positive health impact [29]. We stress that it is important to also look for negative effects, which were part of the inclusion criteria used in our review, although we did not find any reported harms. It is also important that robust evaluation methods [30] are used.

The studies identified in this review were all conducted at very early stages of the PHR adoption life cycle. The systems had often been newly developed, and were being trialled in new users. The longest studies ran for 12 months, which is arguably not long enough for many of the potential benefits of a PHR to be manifest as personal or clinical outcomes. It is perhaps a general weakness of the discipline of health informatics that evaluations are done very early in the development and deployment of an innovative technology, and that we do not evaluate mature products in use. This might be because it is difficult to obtain academic funding to evaluate something that has left the research workbench, or because once solutions become products there are commercial sensitivities that limit the opportunities for objective research.

In 2010 Greenhalgh et al undertook an in depth multi-modal evaluation of the
NHS electronic personal health record: HealthSpace [31]. The study found that adoption was too low and too early to have yet delivered any clinical care benefit (such as from clinicians reviewing HealthSpace records with their patients).

\section{Conclusion}

This review has identified evidence that personal engagement in maintaining prevention oriented PHRs coupled with care planning tools can increase the uptake of preventive health measures, that active engagement in disease management plans can increase patient commitment to better disease control, and that sharing medication records between providers might save clinician time. PHRs can increase perceptions of the patient centeredness of care.

This review does not identify a lack of evidence for the value of PHRs, but rather the scant investments made to date in seeking for such evidence. An equivalent review might be more successfully repeated in five years time, by which time more outcomes may have been identified. There is still limited evidence on how the use of personally determined health, prevention and lifestyle information can impact on health and wellness. It may therefore also be a problem that we need more time to identify what beneficial effects we should be looking for. However, it may also be that we are seeking to identify benefits from a technology which is an adaptation of a tool that is (just now) proving useful to clinical practice, the electronic health record, but that a somewhat different solution is ideally suited for personally directed health care and wellness management. Greenhalgh et al, concluding their review of NHS HealthSpace, suggest that:

“...unless personal electronic records align closely with people's attitudes, self management practices, identified information needs, and the wider care package (including organisational routines and incentive structures for clinicians), the risk that they will be abandoned or not adopted at all is substantial. As the NHS considers the next question for such records, we suggest that conceptualising them dynamically (as components of a socio-technical network) rather than statically (as containers for data), and applying user centred design principles more explicitly, might improve their chances of adoption and use." [30]

The optimal paradigm of PHRs might not mirror that of EHRs - being individual centric - but might be more successful by being family or community centric i.e. their social networking effect might be of greater value than the value of their information content. This vision of a socio-technical PHR network goes beyond the ISO 14292 definition used for this literature review, and may need to be included in the scope of future definitions.

\section{References}

1. https://healthy.kaiserpermanente.org/health/care/ consumer/my-health-manager (accessed January 2013)

2. https://www.myhealth.va.gov/index.html (accessed January 2013)

3. The European Commission. Strategic implementation plan for the European Innovation Partnership on active and healthy ageing. November 2011, Brussels. Available from: http://ec.europa.eu/ research/innovation-union/pdf/active-healthy-ageing/steering-group/implementation_plan.pdf (accessed January 2013)

4. Please see http://www.nhs.uk/NHSEngland/AboutNHSservices/doctors/Pages/expert-patients-programme.aspx (accessed January 2013)

5. U.S. Personal Health Records Market 2010 to 2015. Frost \& Sullivan Research. Summarised at http://www.frost.com/prod/servlet/report-brochure.pag?id=N98D-01-00-00-00 (accessed January 2013)

6. Connecting for Health. Connecting Americans to their healthcare. Final report of the working group on policies for electronic information sharing between doctors and patients. New York: Markle Foundation; 2004. http://www.markle.org/health/ publications-briefs-health/1250-connecting-americans-their-health-care (accessed January 2013).

7. Tang P, Ash J, Bates D, Overhage M, Sands D. Personal health records: definitions, benefits, and strategies for overcoming barriers to adoption. J Am Med Inform Assoc 2006;13:121-6.

8. Pagliari C, Detmer D, Singleton P. Potential of electronic personal health records. BMJ 2007;335:330-3.

9. International Organization for Standardization. ISO TR 14292. Personal Health Records - Definition, Scope and Context. Geneva; 2011.

10. International Organization for Standardization. ISO 18308. Requirements for an Electronic Health Record Architecture. Geneva; 2010. 
11. "Health is a state of complete physical, mental and social well-being and not merely the absence of disease or infirmity." WHO. Preamble to the Constitution of the World Health Organization as adopted by the International Health Conference, New York, 19-22 June 1946, and entered into force on 7 April 1948. Available from: http://www.who. int/governance/eb/who_constitution_en.pdf

12. Long T-Y. Toward patient-centered, personalised and personal decision support and knowledge management: a survey. Yearb Med Inform 2012:104-12.

13. Please see: http://cordis.europa.eu/fp7/ict/ programme/challenge5_en.html (accessed December 2012)

14. Rigby M. Personal health, person centred health and personalised medicine - concepts, consumers, confusion and challenges in the informatics world. Yearb Med Inform 2012:7-15.

15. Kalra D, Fernando B, Morrison Z, Sheikh A. A review of the empirical evidence of the value of structuring and coding of clinical information within electronic health records for direct patient care. [In press]

16. Kim J, Jung H, Bates D. History and Trends of "Personal Health Record" Research in PubMed. Healthc Inform Res 2011 March;17(1):3-17.

17. Cochrane Effective Practice and Organisation of Care Group. Please see http://epoc.cochrane.org/

18. Institute of Medicine. Crossing the quality chasm: A new health systems for the 21 st Century. National Academy Press; 2001.

19. Nagykaldi Z, Aspy C, Chou A, Mold J. Impact of a wellness portal on the delivery of patient-cen- tered preventive care. J Am Board Fam Med 2012;25:158-67.

20. Wagner PJ, Dias J, Howard S, Kintziger KW, Hudson MF, SeolYH, et al. Personal health records and hypertension control: a randomized trial. J Am Med Inform Assoc 2012;19:626-34.

21. Lau A, Sintchenko V, Crimmins J, Magrabi F, Gallego B, Coiera E. Impact of a web-based personally controlled health management system on influenza vaccination and health services utilization rates: a randomized controlled trial. J Am Med Inform Assoc 2012;19:719-27.

22. Grant RW, Wald JS, Schnipper JL, Gandhi TK, Poon EG, Orav EJ, et al. Practice-linked online personal health records for type 2 diabetes mellitus. Arch Intern Med 2008;168(16):1776-82.

23. Kim E, Mayani A, Modi S, Kim Y, Soh C. Evaluation of a patient-centered electronic health record to overcome the digital divide. Conf Proc IEEE Eng Med Biol Soc 2005;1:593-6.

24. Gysels M, Richardson A, Higginson I. Does the patient-held record improve continuity and related outcomes in cancer care: a systematic review. Health Expect 2006;10:75-91.

25. Archer N, Fevrier-Thomas U, Lokker C, McKibbon K, Straus S. Personal health records: a scoping review. J Am Med Inform Assoc 2011;18:515-22.

26. Schnipper JL, Gandhi TK, Wald JS, Grant RW, Poon EG, Volk LA, et al. Effects of an online personal health record on medication accuracy and safety: a cluster-randomised trial. J Am Med Inform Assoc 2012;19(5):728-34.

27. Turvey CL, Zulman DM, Nazi KM, Wakefield BJ, Woods SS, Hogan TP, et al. Transfer of Information from Personal Health Records: A Survey of Vet- erans Using My HealtheVet. Telemed J E Health 2012 Mar; 18(2):109-14.

28. Tenforde M, Nowacki A, Jain A, Hickner J. The Association Between Personal Health Record Use and Diabetes Quality Measures. J Gen Intern Med 2011;27(4):420-4.

29. Krogsbøll L, Juhl K, Larsen C, Gøtzsche P. General health checks in adults for reducing morbidity and mortality from disease: Cochrane systematic review and meta-analysis. BMJ 2012;345:e7191.

30. Friedman CP, Wyatt J. Evaluation Methods in Biomedical Informatics (Health Informatics). Springer, 2010

31. Greenhalgh T, Hinder S, Stramer K, Bratan T, Russell J. Adoption, non-adoption, and abandonment of a personal electronic health record: case study of HealthSpace. BMJ 2010;341:c5814.

Correspondence to:

Dipak Kalra

Clinical Professor of Health Informatics

Director, Centre for Health Informatics

and Multiprofessional Education

University College London

Holborn Union Building, Highgate Hill,

London N19 5LW, United Kingdom

President, The EuroRec Institute

Honorary Consultant, The Whittington Hospital NHS Trust,

London, United Kingdom

Tel: + 442072885966

E-mail:d.kalra@ucl.ac.uk 